\title{
Toxicity Mechanisms and Potential Uses of Extracted Mushroom Toxins - Amatoxin, Antamanide, Muscurine
}

\author{
Xiaochen Liang ${ }^{1, a^{*}, \dagger}$, Tianxu Wang ${ }^{2, b^{*}, \dagger}$ \\ ${ }^{1}$ The University of British Columbia, Vancouver, British Columbia, V6T 1Z1, Canada \\ ${ }^{2}$ Monash University, Monash, Victoria 3168, Australia \\ $\dagger$ These authors contributed equally.
}

\begin{abstract}
Mushroom, as a popular kind of wild edible fungi, has raised the attention of many scientists around the globe, due to the fact that increasing numbers of fatal mushroom poisoning cases worldwide have been reported each year. Therefore, to overcome the issue of fatality of mushrooms with an attempt to help people prevent mushroom poisoning, studying mushroom toxicology has become a significant topic to many biochemists; a growing number of researches are conducted focusing on mushroom toxins as well as their chemical properties and toxicity mechanism. In this review, properties and mechanisms of three major toxins (amatoxin, antamanide, muscarine) are chosen as the research focus. The three major toxins, which are extracted from wild mushrooms, will be summarized and studied based on past research findings. Our goals are trying to find possible treatments of each toxin as well as their potential usage in the pharmaceutical industry, which can be especially beneficial towards research of mushroom-extracted drug and food-poisoning toxicity treatment for further investigation and academic references.
\end{abstract}

\section{Introduction}

Mushroom poisoning has been a severe problem around the globe for a long history [1]. In the US, for the past eighteen years, more than thirteen-thousand cases of mushroom exposure have been reported, including 704 cases that had caused major harm [2]. In France, from 2010 to 2017, around ten thousand cases and 22 deaths had been recorded, which is approximately 1325 cases a year [3]. In China, 276 cases in 17 provinces had been reported in 2019, including 22 deaths a year. As we can see from the data, mushroom poisoning has been an outstanding global issue; therefore, many scientists have been trying to find out the toxins in mushrooms that can lead to major harm for centuries. In our review, we mainly aim to clarify the recent progress on such a study topic by summarizing and organizing the chemical properties and toxicology mechanisms of three major toxins, amatoxin, antamanide, muscarine, which are extracted from Amanita phalloides and Amanita muscaria. Based on past research and experimental data, possible treatment and usage will be further investigated.

\section{Overview of Mushroom Toxicology}

Mushroom toxins can be classified into six different groups [4], which are cytotoxic, neurotoxic, myotoxic, metabolic, gastrointestinal irritant and miscellaneous adverse reactions. These types of toxins can have varied syndromes. From past medical examinations, the most common syndromes of mushroom poisoning are vomiting, incontinence, gastrointestinal irritants, prompt kidney damage, dizziness, fever, unclear vision and diarrhea (As shown in Figure 1.).

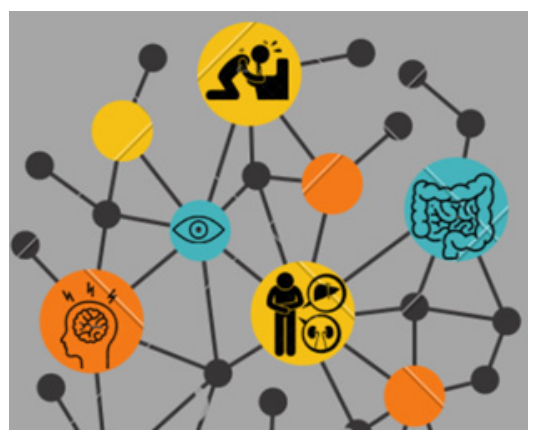

Fig. 1. Main syndromes from mushroom poisoning.

Specifically, the three main toxins that are discussed in this review are derived from the Genus Amanita, in which it contains hundreds of agarics and the most toxic mushrooms at present. These toxins demonstrate a common structure of cyclic-peptides, which includes mono-cyclo and bi-cyoclo. These characteristics, in return, contribute to their toxicity mechanism. Further details will be summarized in the following sections.

\subsection{Amatoxins}

\subsubsection{General Information}

The first type of toxin is the amatoxins. It is presented mainly in three types of species: Amanita, Galerina, and

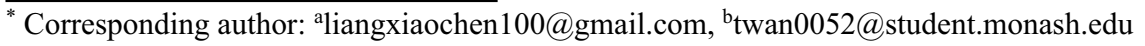


Lepiota. There had been many studies indicating that amatoxin-containing mushrooms will result in fatal mushroom poisonings. The LD50 of amatoxins in mice is $0.3-20 \mathrm{mg} / \mathrm{kg}$. The estimated median lethal dose in humans is $0.1 \mathrm{mg} / \mathrm{kg}$. An unequal distribution of the toxins exists in mushrooms, and in Amanita species for example, the highest content was found in the ring, gills, and the caps [5].

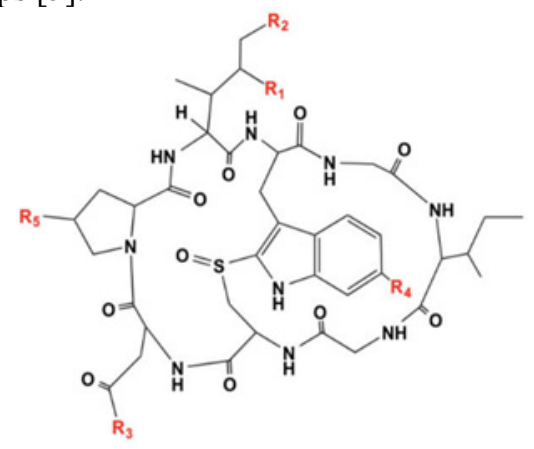

Fig. 2. Amatoxin [4]

Table 1. Amatoxin Compound Chart [4]

\begin{tabular}{llllll}
\hline & R1 & R2 & R3 & R4 & R5 \\
\hline Phalloidin & $\mathrm{OH}$ & $\mathrm{H}$ & $\mathrm{CH}_{3}$ & $\mathrm{CH}_{3}$ & $\mathrm{OH}$ \\
Phalloin & $\mathrm{H}$ & $\mathrm{H}$ & $\mathrm{CH}_{3}$ & $\mathrm{CH}_{3}$ & $\mathrm{OH}$ \\
Prophallin & $\mathrm{H}$ & $\mathrm{H}$ & $\mathrm{CH}_{3}$ & $\mathrm{CH}_{3}$ & $\mathrm{H}$ \\
Phallisin & $\mathrm{OH}$ & $\mathrm{OH}$ & $\mathrm{CH}_{3}$ & $\mathrm{CH}_{3}$ & $\mathrm{OH}$ \\
Phallacin & $\mathrm{H}$ & $\mathrm{H}$ & $\mathrm{CH}_{(}\left(\mathrm{CH}_{3}\right)_{2}$ & $\mathrm{COOH}$ & $\mathrm{OH}$ \\
Phallacidin & $\mathrm{OH}$ & $\mathrm{H}$ & $\mathrm{CH}\left(\mathrm{CH}_{3}\right)_{2}$ & $\mathrm{COOH}$ & $\mathrm{OH}$ \\
Phallisacin & $\mathrm{OH}$ & $\mathrm{OH}$ & $\mathrm{CH}\left(\mathrm{CH}_{3}\right)_{2}$ & $\mathrm{COOH}$ & $\mathrm{OH}$ \\
\hline
\end{tabular}

Amatoxins are identified as bicyclic octapeptides, containing at least nine sub compounds, including alpha amanitin, beta amanitin, gamma amanitin, etc. These are classified into neutral and acidic amatoxins. In this review paper, we are going to mainly consider the alpha amanitin. These compounds only differ from their number of hydroxyl groups and amide carboxyl exchange (Shown in Amatoxin Compound Chart). The reason why they are extremely toxic is because of their great heat stability and solubility. They cannot be broken down by either enzymes or acids. This means that they cannot be destroyed by food processing or gastrovascular digestion [6].

\subsubsection{Toxicity Mechanism}

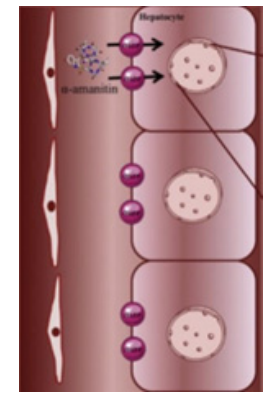

Fig. 3. Uptake in Liver [4]

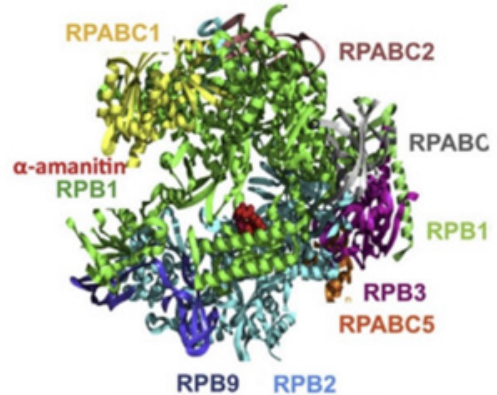

Fig. 4. 3D Structure of RNA Polymerase 2

Liver is the first organ affected after injection. The absorption of the toxin is by the OATP in the sinusoidal membrane of hepatocytes. They do not undergo metabolism in the human body and a large amount of it will be finally excreted in the urine $[6,7]$. There are several different kinds of mechanisms. The most common one seems to be the covalent binding to RNA Polymerase 2 . The binding site is on the connecting site of Rpb1 and Rpb2 of RNAP 2 [7].

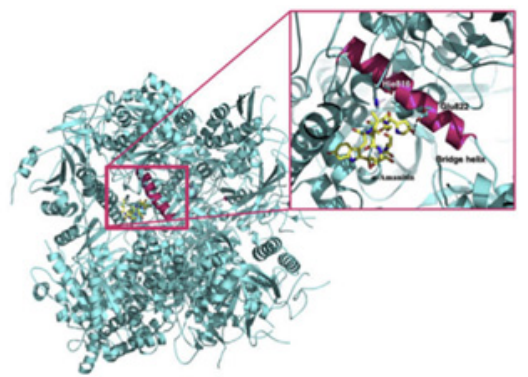

Fig. 5. The Binding site of Amanitin Alpha

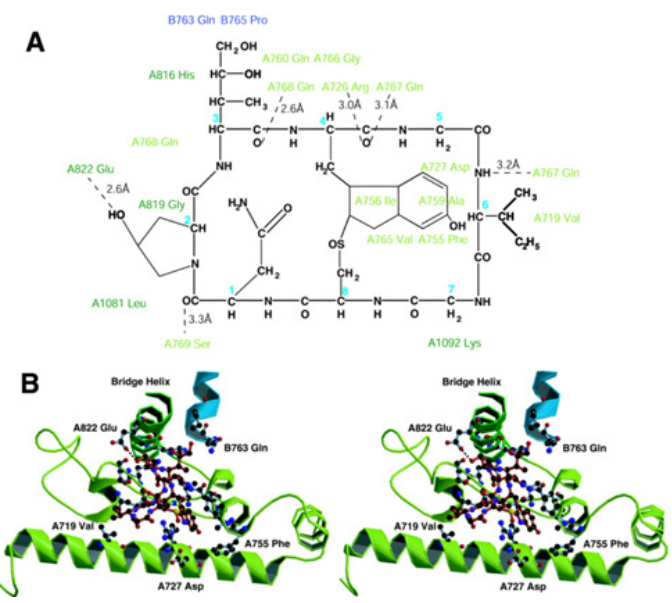

Fig. 6 Binding site of Rpb1 and Rpb2 of RNAP 2 [7]

Their interactions are almost located entirely in the "bridge helix" [7], including 1) Hydroxyproline 2 binding with Glu-A822; 2) Carboxyl group of 4,5dihydroxyisoleucine 3 with Gln-A768, binding with HisA816; 3) Alpha amanitin with region of Rpb1 near the bridge helix. This leads to an inhibition of RNA Polymerase, which reduces the speed of translocation along DNA. Also, many scientists also argued that alpha amanitin can interact with the trigger loop of RNAP 2, affecting the RNA elongation process. This causes the reduced speed of protein synthesis, and potentially causes cell death [4]. 
Alpha amanitin poisoning may cause severe liver injuries, and some in vitro studies also proposed that apoptosis may occur. Studies showed that p53 is affected by alpha amanitin. There are also interactions between p53 and mitochondrial BAK, that is important to alpha amanitin related apoptosis.

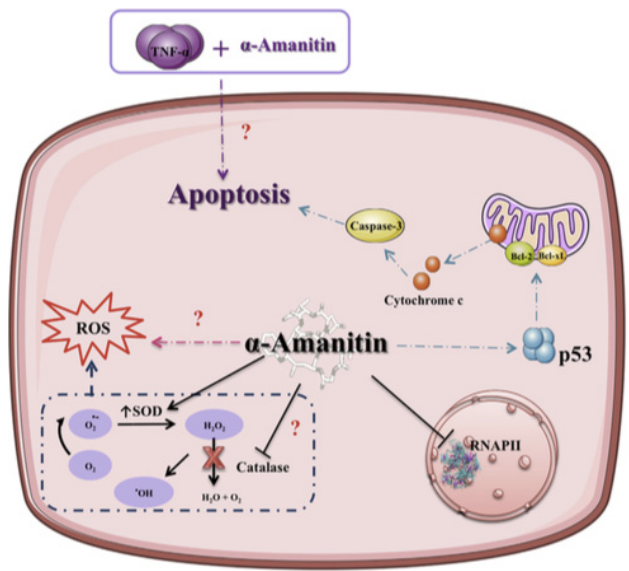

Fig. 7. Other Mechanisms of Alpha Amanitin [4]

Other mechanisms include tumor necrosis factor alpha, aggravating alpha amanitin induced hepatotoxicity. This can also lead to potential hepatocyte apoptosis. TNF alpha co-treatments can increase lipid peroxidation that is caused by alpha amanitin, resulting in a link between TNF alpha induced toxicity with reactive oxygen species production. Accumulated alpha amanitin also causes additional superoxide dismutase, and a lower catalase activity. In fact, alpha amanitin can form phenoxyl free radicals that are potentially involved in ROS generation. However, this still needs further investigation.

\subsection{Antamanide (AA)}

\subsubsection{General Information}

AA is commonly found in Amanita, as shown in Figure 8 , which is the same source as amatoxin. Amanita has attracted the attention of many chemists and biochemists and has been intensively studied, due to the fact that such a type of mushroom is one of the most dangerous species currently known, causing $90 \sim 95 \%$ of all deaths from mushroom poisoning (Yin et al., 2019). However, even though AA is from the same source as amatoxins, it is non-toxic to animals. Interestingly, they are very similar chemicals which are both cyclo-peptides.

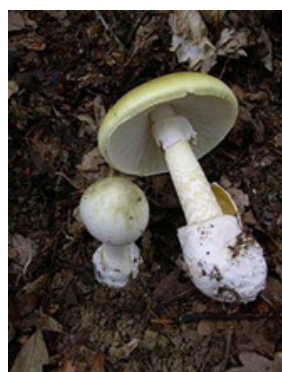

Fig. 8. Amanita (death cap)

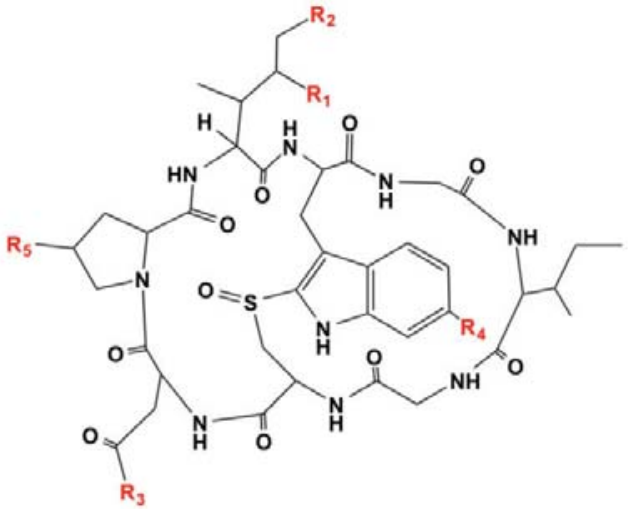

(a)

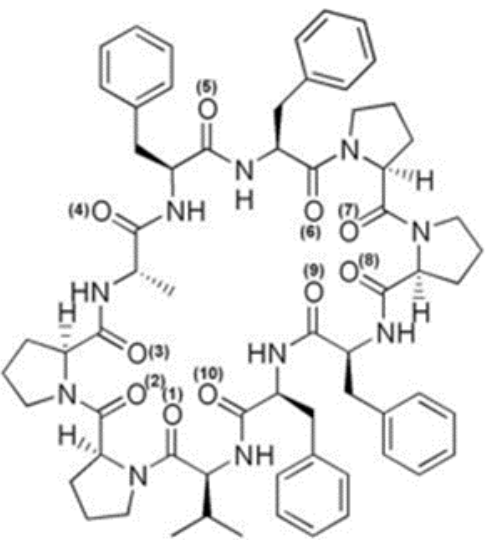

(b)

Fig. 9. Comparison Between Amatoxin (a) \& AA (b)

Amatoxin is bi-cyclopeptides, and AA is monocyclopeptides. The side groups in AA are proline, phenylalanine, alanine, and valine, as illustrated in Figure 10.

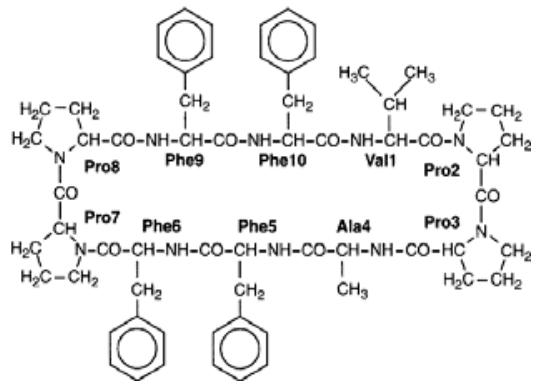

Fig. 10. Chemical Structure of AA

\subsubsection{Toxicity Mechanism}

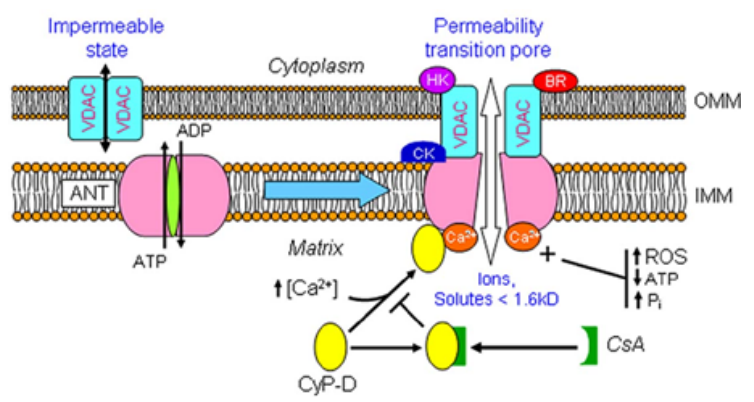

Fig. 11. Permeability Transition Pore

AA inhibited the mitochondrial permeability transition pore (PTP) by targeting the pore regulator cyclophilin D 
[8]. PTP is a transmembrane protein residing in the mitochondrial inner membrane. Normally closed, but will only open when the matrix is accumulated with calcium ions, and such a process is related to apoptosis. Once the PTP is opened, apoptotic factors will be released into the cytoplasm, which will lead to apoptosis. Based on this mechanism, an experiment (Whitworth, J. 2019) had been conducted, and the results are shown in Figure 12.

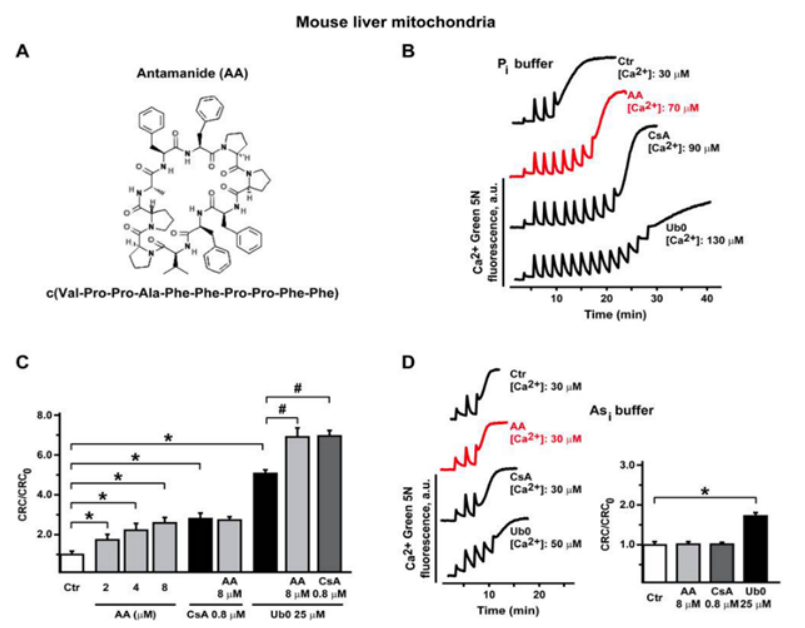

Fig. 12. Experimental Data \& Plots

To evaluate the effect of AA on PTP, a calcium ion retention capacity assays on isolated mouse liver mitochondria is performed, and two mediums have been tested. The first medium is in phosphate containing buffer, which is in B (Pi buffer), and the second one is in arsenate buffer $\mathrm{D}$ (As i buffer). The reason why two sets are performed is because when mitochondria is incubated in a phosphate environment, AA inhibits pore opening, which is similar to other known inhibitors such as CsA and Ub0. To compare, the arsenate environment is set to see the difference under different environments.

In $\mathrm{B}$ and $\mathrm{D}$, the $\mathrm{X}$-axis is time in minutes, and the $\mathrm{Y}$ axis is the calcium green fluorescence in a.u. Unit. The overall increased trends in both graphs ( $B$ and $D)$ is caused by AA, CsA and Ub0 that act as pore inhibitors and increase the threshold calcium ions concentration required to trigger the permeability transition. And such data proves that $\mathrm{AA}$ is an inhibitor that causes the toxicity to human bodies.

\subsection{Muscarine}

\subsubsection{General Information}

Muscarine is a water-soluble toxin which was discovered through isolation of the mushroom species Amanita muscaria shown in figure 13, and this mushroom is easily distinguished as toxic.

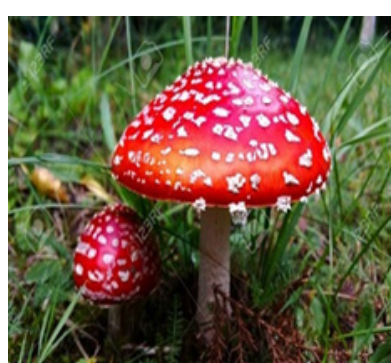

Fig. 13. Amanita muscaria

However, the relative high concentration of this natural toxin can be found in mushroom species called Inocybe geophylla, as shown in Figure 14, and this mushroom is particularly widespread in North America and Europe.

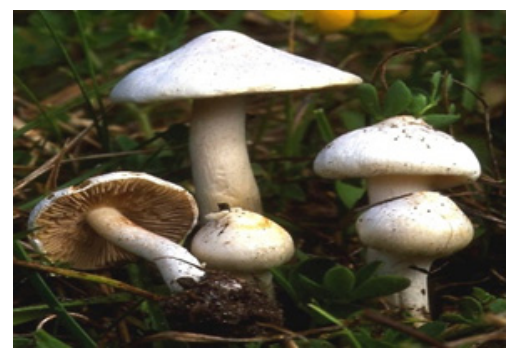

Fig. 14. Inocybe geophylla

Different from the AA toxin, muscarine is poisonous for both humans and animals, according to the paper [9], dogs are particularly susceptible to muscarine poisoning with many cases finally resulting in death. The general symptoms, which include profuse sweating, muscular cramps, and bradycardia (heart rate that's too slow), will start quickly within two hours after the consumption of the mushrooms [10].

\subsubsection{Toxicity Mechanism}

The mechanism of toxicity of muscarine is relatively straightforward. Muscarine could mimic the function of the natural neurotransmitter acetylcholine in the muscarinic part of the nervous system and compete with acetylcholine at its receptor binding site which is called muscarinic acetylcholine receptors [11]. Figure 15 demonstrates how acetylcholine binds to muscarinic acetylcholine receptors at normal condition.

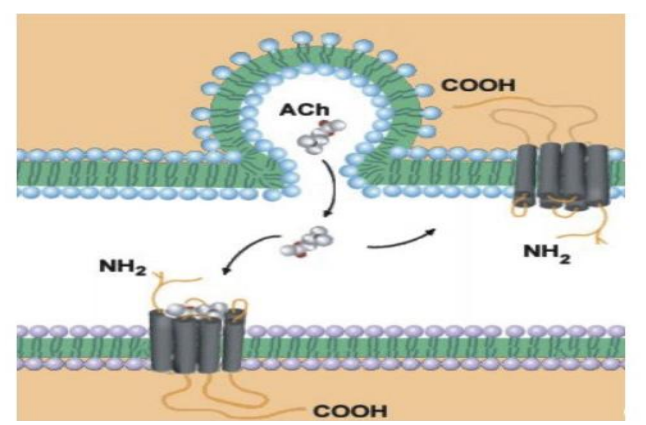

Fig. 15. Acetylcholine binds to muscarinic acetylcholine receptors at normal condition. 
Figure 16-18 present the structures of muscarine and acetylcholine. From their chemical structures it can be found the reason why muscarine could mimic the function of acetylcholine is that they have similar chemical groups.

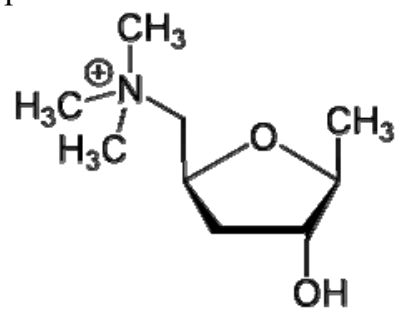

Fig. 16. 2D structure of muscarine.

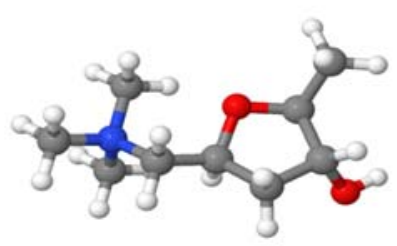

Fig. 17. 3D structure of muscarine<smiles>CC(=O)OCC[N+](C)(C)C</smiles>

Fig. 18. Structure of acetylcholine

It is also essential to know why muscarine mimic acetylcholine could be harmful, Acetylcholine is the primary neurotransmitter of the sympathetic and parasympathetic nervous system, which controls smooth muscle contraction, blood vessel dilation and slows heart rate, as can be seen in Figure 19. Therefore, when the foreign toxin (muscarine) gets into human body, it is not controllable as acetylcholine, which is synthesized in cytoplasm of nerve terminals and results in the disruption of body mechanism.

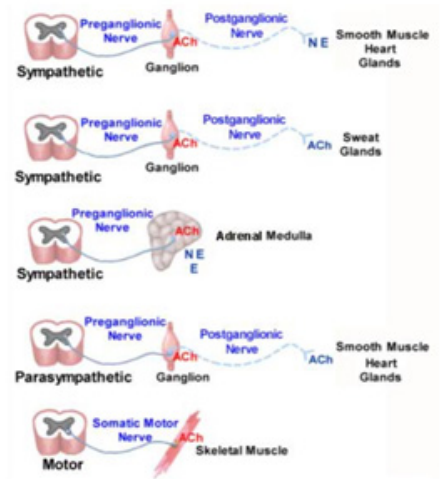

Fig. 19. Peripheral and autonomic sites where $\mathrm{ACh}$ is neurotransmitter

Muscarinic acetylcholine receptors belong to the family of G-protein-coupled receptors (GPCR). They are involved in the various autonomic physiological functions of the human body. G-Protein coupled receptors (GPCRs) are a group of seven transmembrane proteins which bind to signal molecules outside of the cell, and then transduce the signal into the cell and finally cause different cellular responses such as apoptosis, activation of protein kinase etc. The GPCRs work with the help of a G-Protein which will be activated like the figure shows, binds to the energy rich GTP and kicks off GDP. The activation of GTP could result in complicated cascade reactions.

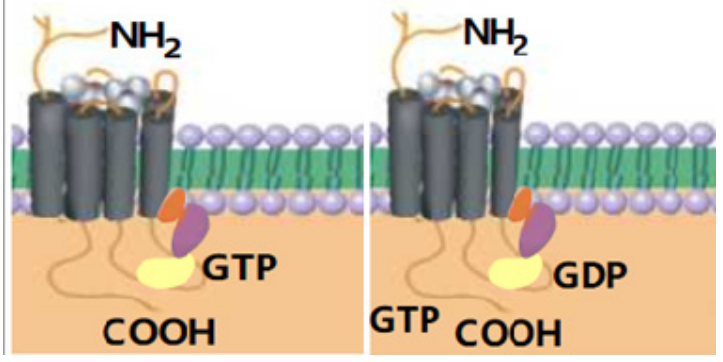

Fig. 20. Subunit of the G protein releases bound GDP and simultaneously binds GTP

\section{Conclusion}

In summary, we generated and analyzed data from multiple current studies to conclude possible treatments toward each toxin. However, For Amatoxins and AA toxin, collecting results from several studies, there are no conclusive available treatments other than liver transplant, therefore further studies and investigations are needed. For muscarine, because of its simple principle, most patients with muscarinic toxin can be treated with atropine, which could inhibit the effects of muscarine: more specifically, atropine could inhibit muscarine bind to acetylcholine binding site. Therefore, atropine could efficiently decrease symptoms like excessive fluid secretions and bradycardia. The possible treatment in the future will need to consider different toxin mechanism in these specific types of mushrooms to develop treatment strategies accordingly.

\section{References}

1. Brandenburg, W. E. (2018, July 31). Mushroom poisoning epidemiology in the United States. Retrieved September 24, 2020, from https:/www.tandfonline.com/doi/abs/10.1080/0027 5514.2018.1479561? journalCode=umyc20

2. Whitworth, J. (2019, December 18). Thousands poisoned by mushrooms in France in recent years. Retrieved September 24, 2020, from https://www.foodsafetynews.com/2019/12/thousand s-poisoned-by-mushrooms-in-france-in-recentyears/

3. Li, H., Zhang, H., Zhang, Y., Zhang, K., Zhou, J., Yin, Y., . . S Sun, C. (2020, January 01). Mushroom Poisoning Outbreaks - China, 2019. Retrieved September 24, 2020, from http://weekly.chinacdc.cn/en/article/doi/10.46234/cc dcw2020.005 
4. Yin, X., Yang, A., \&amp; Gao, J. (2019). Mushroom Toxins: Chemistry and Toxicology. Journal of Agriculture and Food Chemistry, 67(5053), 5071st ser., 5053-5071. doi:10.1021/acs.jafc.9b00414

5. Diaz, J. H. (2018). Amatoxin-Containing Mushroom Poisonings: Species, Toxidromes, Treatments, and Outcomes. Wilderness \& Environmental Medicine, 29(1),

111-118. https://doi.org/10.1016/j.wem.2017.10.002

6. Garcia, J., Costa, V. M., Carvalho, A., \& Baptista, P. (2015). Amanita phalloides poisoning: Mechanism of toxicity and treatment. Food and Chemical Toxicity, 86, 41-55. https://doi.org/10.1016/j.fct.2015.09.008

7. Bushnell, D. A., Cramer, P., \& Kornberg, R. D. (2002). Structural basis of transcription: -AmanitinRNA polymerase II cocrystal at 2.8 A resolution. Proceedings of the National Academy of Sciences, 99(3),

$1218-1222$. https://doi.org/10.1073/pnas.251664698

8. Azzolin, L., Antolini, N., Calderan, A., Ruzza, P., Sciacovelli, M., Marin, O., . . Rasola, A. (2011, January 28). Antamanide, a derivative of Amanita phalloides, is a novel inhibitor of the mitochondrial permeability transition pore. Retrieved September 24, 2020, from https://www.ncbi.nlm.nih.gov/pmc/articles/PMC303 0572/

9. Stansfield, W., Ranek, M., Pendse, A., Schisler, J., Wang, S., Pulinilkunnil, T., \&amp; Willis, M. (2014, January 02). The Pathophysiology of Cardiac Hypertrophy and Heart Failure. Retrieved September 25, 2020, from https:/www.sciencedirect.com/science/article/pii/B9 780124052062000041

10. Kosentka, P., Sprague, S. L., Ryberg, M., Gartz, J., May, A. L., Campagna, S. R., \& Matheny, P. B. (2013). Evolution of the Toxins Muscarine and Psilocybin in a Family of Mushroom-Forming Fungi. PLoS ONE, 8(5), e64646. https://doi.org/10.1371/journal.pone.0064646

11. Acetylcholine Neurotransmission (Section 1, Chapter 11) Neuroscience Online: An Electronic Textbook for the Neurosciences | Department of Neurobiology and Anatomy - The University of Texas Medical School at Houston. (n.d.). Uth.Tmc.Edu. Retrieved September 24, 2020, from https://nba.uth.tmc.edu/neuroscience/m/s1/chapter 11 .htm 